\title{
SPHERE THEOREM FOR MANIFOLDS WITH POSITIVE CURVATURE
}

\author{
BAZANFARÉ MAHAMAN \\ Chef du Département de Mathématiques and Informatique Faculté des Sciences, BP 10662 Niamey-Niger \\ e-mail:bmahaman@yahoo.fr
}

(Received 16 February, 2005; accepted 10 October, 2005)

\begin{abstract}
In this paper, we prove that, for any integer $n \geq 2$, and any $\delta>0$ there exists an $\epsilon(n, \delta) \geq 0$ such that if $M$ is an $n$-dimensional complete manifold with sectional curvature $K_{M} \geq 1$ and if $M$ has conjugate radius $\rho \geq \frac{\pi}{2}+\delta$ and contains a geodesic loop of length $2(\pi-\epsilon(n, \delta))$ then $M$ is diffeomorphic to the Euclidian unit sphere $\mathbb{S}^{n}$.
\end{abstract}

2002 Mathematics Subject Classification. 53C20, 53C21.

1. Introduction. One of the fundamental problems in Riemannian geometry is to determine the relation between the topology and the geometry of a Riemannian manifold. In this way the Toponogov's theorem and the critical point theory play an important role. Let $M$ be a complete Riemannian manifold and fix a point $p$ in $M$ and define $d_{p}(x)=d(p, x)$. A point $q \neq p$ is called a critical point of $d_{p}$ or simply of the point $p$ if, for any nonzero vector $v \in T_{q} M$, there exists a minimal geodesic $\gamma$ joining $q$ to $p$ such that the angle $\measuredangle\left(v, \gamma^{\prime}(0)\right) \leq \frac{\pi}{2}$. Suppose $M$ is an $n$-dimensional complete Riemannian manifold with sectional curvature $K_{M} \geq 1$. By Myers' theorem the diameter of $M$ is bounded from above by $\pi$. In [4] Cheng showed that the maximal value $\pi$ is attained if and only if $M$ is isometric to the standard sphere. It was proved by Grove and Shiohama [5] that if $K_{M} \geq 1$ and the diameter of $M \operatorname{diam}(M)>\frac{\pi}{2}$ then $M$ is homeomorphic to a sphere.

Hence the problem of removing homeomorphism to diffeomorphism or finding conditions to guarantee the diffeomorphism is of particular interest. In [13] C. Xia showed that if $K_{M} \geq 1$ and the conjugate radius $\rho(M)$ of $M$ is greater than $\pi / 2$ and if $M$ contains a geodesic loop of length $2 \pi$, then $M$ is isometric to $\mathbb{S}^{n}$.

DEFINITION 1.1. Let $\mathrm{M}$ be an $\mathrm{n}$-dimensional Riemannian manifold and $p$ be a point in $M$. Let $\operatorname{Conj}(p)$ denote the set of first conjugate points to $\mathrm{p}$ on all geodesics issuing from $p$. The conjugate radius $\rho(p)$ of $M$ at $p$ in the sense of Xia [13] is defined as

$$
\rho(p)=d(p, \operatorname{Conj}(p)) \quad \text { if } \operatorname{Conj}(p) \neq \varnothing
$$

and

$$
\rho(p)=+\infty \quad \text { if } \operatorname{Conj}(p)=\emptyset
$$

Then the conjugate radius of $M$ is given by

$$
\rho(M)=\inf _{x \in M} \rho(x) .
$$


Many interesting results have been proved by using the critical points theory and Toponogov's theorem [3], [5], [7], [8], [10], [11], [12], [13]. etc...

The purpose of this paper is to prove the following result.

THEOREM 1.2. For any $n \geq 2$ and any $\delta>0$, there exists a positive constant $\epsilon(n, \delta)$ depending only on $n$ and $\delta$ such that for any $\epsilon \leq \epsilon(n)$, if $M$ is an $n$-dimensional complete connected Riemannian manifold with sectional curvature $K_{M} \geq 1$ and conjugate radius $\rho(M)>\frac{\pi}{2}+\delta$ and if $M$ contains a geodesic loop of length $2(\pi-\epsilon)$ then $M$ is diffeomorphic to an n-dimensional unit sphere $\mathbb{S}^{n}$ and the metric $g$ of $M$ is $\epsilon^{\prime}=\epsilon^{\prime}(\epsilon, n, \delta, \alpha)$ close in the $\mathcal{C}^{\alpha}$ topology to the canonical metric of curvature 1 of $\mathbb{S}^{n}$ for any $\left.\alpha \in\right] 0,1[$.

Proof. Let $i(M)$ denote the injectivity radius of $M$. By definition we have

$$
i(M)=\inf _{x \in M} d(x, C(x))
$$

where $C(x)$ is the set of cut points of $x$.

A classical result due to Klingenberg (see for instance corollary 4.14 of [9]) asserts that if $M$ is compact then $i(M)=\min \left\{t_{0}, \frac{l_{0}}{2}\right\}$, where $l_{0}$ is the minimum of the length of non trivial closed geodesics of $M$ and $t_{0}$ is the minimum over unit vector $u$ of $T M$ of the first conjugate value $t_{0}(u)$ along the geodesic $\gamma_{u}(t)=\exp (t u)$.

LEMMA 2.1. Let $M$ be an n-dimensional complete, connected Riemannian manifold with sectional curvature $K_{M} \geq 1$. With Xia's convention on the conjugate radius we have $i(M) \geq \rho(M)$.

The proof is a direct application of the Klingenberg's result: by the definition above of the conjugate radius we have $t_{0} \geq \rho(M)$ and, since $K_{M} \geq 1$, every geodesic $\gamma$ issued from a point $p$ hits $\operatorname{Conj}(p)$ at a point $q$ (by the Rauch comparison theorem). Consequently, the length of every non trivial closed geodesic issued from $p$ is bounded below by $2 d(p, q) \geq 2 \rho(M)$.

LEMMA 2.2. For any $\delta>0$, there exists a function $\tau_{\delta}$ which satifies $\lim _{\epsilon \mapsto 0} \tau_{\delta}(\epsilon)=0$ and such that if $M$ is a complete manifold with $K_{M} \geq 1$, injectivity radius $i(M) \geq \frac{\pi}{2}+\delta$ and which contains a geodesic loop of length $2(\pi-\epsilon)$ then we have $\operatorname{diam}(M) \geq \pi-\tau_{\delta}(\epsilon)$.

Proof. Let $\gamma$ be a loop with length $2 \pi-2 \epsilon$. Let $x=\gamma(0)=\gamma(2 \pi-2 \epsilon), y=$ $\gamma(\pi / 2+\delta), m=\gamma(\pi-\epsilon)$ and $z=\gamma\left(\frac{3(\pi-\epsilon)}{2}-\delta\right)$

Let

$$
\gamma_{1}=\gamma /\left[0, \frac{\pi}{2}+\delta\right], \quad \gamma_{2}=\gamma /\left[\frac{\pi}{2}+\delta, \pi-\epsilon\right], \quad \gamma_{3}=\gamma /\left[\pi-\epsilon, \frac{3(\pi-\epsilon)}{2}-\delta\right],
$$

and

$$
\gamma_{4}=\gamma /\left[\frac{3(\pi-\epsilon)}{2}-\delta, 2 \pi-2 \epsilon\right]
$$

Then the geodesics $\gamma_{i}$ are minimal. Let $\sigma$ be a minimal geodesic joining $m$ and $x$. Set $\alpha=\measuredangle\left(\sigma^{\prime}(0),-\gamma^{\prime}(\pi-\epsilon)\right)$ and $\beta=\measuredangle\left(\sigma^{\prime}(0), \gamma^{\prime}(\pi-\epsilon)\right)$.

We have $\alpha \leq \pi / 2$ or $\beta \leq \pi / 2$. Suppose, without loss of generality, that $\alpha \leq \pi / 2$. Applying the Toponogov comparison theorem on length to the hinge formed by $\gamma_{2}$ 
and $\sigma$ at $\gamma(\pi-\epsilon)$ we have

$$
\cos \left(\frac{\pi}{2}+\delta\right) \geq \cos L(\sigma) \cos \left(\frac{\pi}{2}-\epsilon-\delta\right)+\cos \alpha \sin L(\sigma) \sin \left(\frac{\pi}{2}-\epsilon-\delta\right)
$$

so that

$$
\cos L(\sigma) \leq-\frac{\sin \delta}{\sin (\delta+\epsilon)} \Rightarrow L(\sigma) \geq \pi-\tau_{\delta}(\epsilon)
$$

and the conclusion follows.

Note that Anderson [1] and Otsu [6] constructed, for $n \geq 4 n$-dimensional closed manifolds with Ric $\geq n-1$ and diameter arbitrarily close to $\pi$ but whose homotopy type is distinct from that of the sphere. Thus additional assumptions are needed.

In [2] G. Pacelli Bessa proved the following theorem from which we deduce Theorem 1.2.

THEOREM 2.3. Given $n \geq 2$ and $i_{0}>0$ there exists an $\epsilon=\epsilon\left(n, i_{0}\right)$ such that if $M$ admits a metric $g$ satisfying

$$
\text { Ric } \geq n-1, \quad i(M) \geq i_{0}, \quad \operatorname{Diam}(M) \geq \pi-\epsilon
$$

then, for any $\alpha \in] 0,1\left[, M\right.$ is diffeomorphic to $\mathbb{S}^{n}$ and the metric $g$ of $M$ is $\epsilon^{\prime}=\epsilon^{\prime}(\epsilon, n, \alpha)$ close in the $\mathcal{C}^{\alpha}$ topology to the canonical metric of curvature 1 of $\mathbb{S}^{n}$, where $\epsilon^{\prime}$ tends to 0 with $\epsilon$.

REMARK. The complex projectif space shows that theorem 1.2 is false under the weaker hypothesis $\rho \geq \frac{\pi}{2}$.

ACKNOWLEDGEMENT. I would like to thank the referee for his/her insightful and helpful comments.

\section{REFERENCES}

1. M. T. Anderson, Metrics of positive Ricci curvature with large diameters, Mannscripta Math. 68 (1990), 405-415. $245-259$.

2. G. P. Bessa, Differentiable sphere theorems for Ricci curvature, Math. Z. 214 (1993),

3. J. Cheeger, Critical points of distance functions and applications to geometry, in Geometric topology: recent devlopments (Montecafini Terme, 1990), Lectures Notes in Mathematics, No. 1504 (Springer-Verlag, 1991), 1-38.

4. S. Y. Cheng, Eigenvalue comparison theorem and geometric applications, Math. Z. 143 (1975), 289-297.

5. K. Grove and K. Shiohama, A generalized sphere theorem, Ann. of Math. (2) 106 (1977), 201-211.

6. Yukio Otsu, On manifolds of positive Ricci cunvature with Carge diameter, Math. Z. 206 (1991), 255-264.

7. P. Pertersen, Comparison geometry problem list, in Riemannian geometry, Waterloo, ON, 1993 (Amer. Math. Soc., Providence, RI 1996), 87-115.

8. K. Shiohama, A sphere theorem for manifolds of positive Ricci curvature, Trans. Amer. Math. Soc. 275, No. 2 (1983), 811-819. 1996).

9. T. Sakai, Riemannian geometry, Transl. Math. Monographs No. 149 (Amer. Math. Soc., 
10. Z. Shen, Complete manifolds with nonnegative Ricci curvature and large volume growth, Invent. Math. 125 (1996), 393-404.

11. J. Sha and Z. Shen, Complete manifolds with nonnegative curvature and quadratically nonnegative curved infinity, Amer. J. Math. 119 (1997), 1399-1404.

12. C. Xia, Complete manifolds with sectional curvature bounded below and large volume growth, Bull. London Math. Soc. 34 (2002), 229-235.

13. C. Xia, Some applications of critical point theory of distance functions on Riemannian manifolds, Composition Math. 132 (2002), 49-55. 